\title{
On Information Theoretic Games for Interference Networks
}

\author{
Suvarup Saha and Randall A. Berry \\ Dept. of EECS \\ Northwestern University \\ e-mail: \\ suvarups@u.northwestern.edu \\ rberrydeecs.northwestern.edu
}

\begin{abstract}
The Nash equilibrium region for a 2-user game was defined and characterized first for a linear deterministic channel and then for a Gaussian channel. Challanges in extending this understanding to some special $K$-user cases have also been explored. In this paper, we study two indices which compare the performance (sum-rate) of the 'best' and the 'worst' Nash equilibria to the optimum (sum-capacity) and reflect the 'price of stability' and the 'price of anarchy', respectively. These indices are evaluated for the 2 -user and some special $K$-user linear deterministic interference channels. We further investigate the impact on these indices of changing the payoff functions of each user to include a cost of transmission.
\end{abstract}

\section{INTRODUCTION}

Interference has been traditionally viewed as a limiting factor in both wireless and wireline communication systems. With the adoption of new wireless devices, the need for interference management is even more acute. A challenge in effective managing interference is that the self-interest of individual users may not be well aligned. Each user (transmitter-receiver pair) is essentially interested in improving its own performance (e.g. its reliable transmission rate) regardless of the impact of the transmission on other users. Game theory provides a framework for studying such situations.

A natural abstraction of such a situation is to consider a game where a set of users share an interference channel. Each user communicates an independent message over a point-topoint link, and the links interfere with each other through cross-talk.The canonical information theoretic model for an interference channel is the Gaussian interference channel. The capacity region of this channel is not known in general. However, recently the capacity region for the 2-user interference channel has been characterized to within one bit for all values of channel parameters [1]. Furthermore, it is shown in [2] that the high SNR behavior of the 2-user Gaussian interference channel is in fact captured by a linear deterministic interference channel, for which the capacity region can be computed exactly using the results in [4].

In [8], an information theoretic game model for two users communicating over a general interference channel was developed. In particular, the Nash equilibria region of the channel

This work was supported in part by NSF grants CNS-0905407 and CCF0905224 and by the Darpa ITMANET program under the grant W911NF-071-0028. was defined as a natural extension of the information theoretic capacity region. If a pair of rates lie in this region then for long enough block lengths there exists a pair of encoding and decoding strategies from which neither user is willing to deviate if they require arbitrarily small probability of error. In [8], this region was then completely characterized for the 2 -user deterministic interference channel model from [2]. In [10], these results were extended to the 2-user Gaussian interference channel. Beyond two users, the nature of Nash equilibria for a general $K$-user interference channel is unknown, as is the capacity region in general. The Nash equilibria region was partially characterized for some special cases of the $K$-user interference channel was done in [9].

In this paper, we re-examine the information theoretic game in [8] and evaluate the impact of a particular choice of equilibrium on sum-capacity. In particular, we introduce two indices which capture the 'best' and the 'worst' case scenarios possible in an equilibrium from the point-of-view of the sumrate. These indices are evaluated for various symmetric 2 -user and $K$-user interference channels, restricting ourselves to the linear deterministic model. Next we re-formulate the pay-off of each user by introducing a cost for transmission for each level used by a user. Thus, we have a different game, and we restrict ourselves to a family of 'simple strategies' that have been shown to be 'efficient' in [8]. We show that this re-formulation leads to a unique Nash equilibrium for all parameter regimes for the 2-user case, but not so when we have more than two users.

Other game theoretic approaches for interference channels have been studied before, mainly focusing on Gaussian models, e.g. [6], [7]. However, beacuse of the restriction to the use of random Gaussian codebooks or treating the interference as Gaussian noise, the formulation in these works are not information-theoretic in nature. Another approach recently pursued in [11] involves use of dynamic bargaining theory but this also assumes some form co-ordination between the users.

\section{Problem Formulation}

To begin, we recall the definition of the basic interference channel game for $K$ users as introduced in [9]; this directly 
generalizes the $K=2$ user game presented in [8]. Communication starts at time 0 . Each user $i=1, \ldots, K$ communicates by coding over blocks of length $N_{i}$ symbols. Transmitter $i$ sends on block $k$ information bits $b_{i 1}^{(k)}, \ldots, b_{i, L_{i}}^{(k)}$ by transmitting a codeword denoted by $\mathbf{x}_{i}^{(k)}=\left[\mathbf{x}_{i}^{(k)}(1), \ldots, \mathbf{x}_{i}^{(k)}\left(N_{i}\right)\right]$. All the information bits are equally probable and independent of each other. Receiver $i$ observes on each block $k$ an output sequence $\mathbf{y}_{i}^{(k)}=\left[\mathbf{y}_{i}^{(k)}(1), \ldots, \mathbf{y}_{i}^{(k)}\left(N_{i}\right)\right]$ through the interference channel, which specifies a stochastic mapping from the input sequences of users $1, \ldots, K$ to the output sequences of the users. Given the observed sequences up to block $k,\left\{\mathbf{y}_{i}^{(m)}\right\}_{m=1}^{k}$, receiver $i$ generates a guess $\hat{b}_{i \ell}^{(k)}$ for each information bit. Without loss of generality, we assume that this is done via maximum-likelihood decoding on each bit.

Note that this communication scenario is more general than the one usually used in multiuser information theory, as we allow the users to code over different block lengths. Such generality is necessary here, since even though the users may agree $a$ priori on a common block length, a selfish user may unilaterally decide to choose a different block length during the actual communication process.

A strategy $s_{i}$ of user $i$ is defined by its message encoding, which we assume to be the same on every block and involves:

- the number of information bits $L_{i}$ and the block length $N_{i}$ of the codewords,

- the codebook $\mathcal{C}_{i}$ employed by transmitter $i$,

- the encoder $f_{i}:\left\{1, \ldots, 2^{L_{i}}\right\} \times \Omega_{i} \rightarrow \mathcal{C}_{i}$, that maps on each block $k$ the message $m_{i}^{(k)}:=\left(b_{i 1}^{(k)}, \ldots b_{i, L_{i}}^{(k)}\right)$ to a transmitted codeword $\mathbf{x}_{i}^{(k)}=f_{i}\left(m_{i}^{(k)}, \omega_{i}^{(k)}\right) \in \mathcal{C}_{i}$,

- the rate of the code, $R_{i}\left(s_{i}\right)=L_{i} / N_{i}$.

Let $\mathbf{s}=\left(s_{1}, \ldots, s_{K}\right)$ denote a strategy profile, i.e., a choice of strategies for each user $i=1, \ldots, K$. A given profile $\mathbf{s}$ jointly determines the probabilities of error $p_{i}^{(k)}:=$ $\frac{1}{L} \sum_{\ell=1}^{L_{i}} \mathcal{P}\left(\hat{b}_{i \ell}^{(k)} \neq b_{i \ell}^{(k)}\right), i=1, \ldots, K$. Note that if the users use different block lengths, the error probability could vary from block to block even though each user uses the same encoding for all the blocks.

The encoder of each transmitter $i$ may employ a stochastic mapping from the message to the transmitted codeword; $\omega_{i}^{(k)} \in \Omega_{i}$ represents the randomness in that mapping. We assume that this randomness is independent between the two transmitters and across different blocks and is only known at the respective transmitter and not at any of the receivers.

For a given error probability threshold $\epsilon>0$, we define an $\epsilon$-interference channel game as follows. Each user $i$ chooses a strategy $s_{i}$ and receives a pay-off of $\pi_{i}(\mathbf{s})=R\left(s_{i}\right)$ if $p_{i}^{(k)}(\mathbf{s}) \leq \epsilon$, for all $k$; otherwise, $\pi_{i}(\mathbf{s})=0$. In other words, a user's pay-off is equal to the rate of the code provided that the probability of error is no greater than $\epsilon$. A strategy profile $\mathbf{S}$ is defined to be $(1-\epsilon)$-reliable provided that it results in an error probability $p_{i}^{k}(\mathbf{s})$ of less than $\epsilon$ for $i=1, \ldots, K$ and all $k$.

For an $\epsilon$-game, a strategy profile $\mathbf{s}^{*}=\left(s_{1}^{*}, \ldots, s_{K}^{*}\right)$ is a Nash equilibrium (NE) if no single user can unilaterally deviate and improve her pay-off, i.e. if for each user $i=$
$1, \ldots, K$, there is no other strategy $s_{i}$ such that ${ }^{1} \pi_{i}\left(s_{i}, \mathbf{s}_{-i}^{*}\right)>$ $\pi_{i}\left(s_{i}^{*}, \mathbf{s}_{-i}^{*}\right)$. If user $i$ attempts to transmit at a higher rate than what he is receiving in a NE and the other users do not change their strategy, then user $i$ 's error probability must be greater than $\epsilon$. Similarly, a strategy profile $\mathbf{s}^{*}$ ) is an $\eta$-Nash equilibrium ${ }^{2}$ ( $\eta$-NE) of an $\epsilon$-game if no user can unilaterally deviate and improve her pay-off by more than $\eta$, i.e. if for each user $i$, there is no other strategy $s_{i}$ such that $\pi_{i}\left(s_{i}, \mathbf{s}_{-i}^{*}\right)>\pi_{i}\left(s_{i}^{*}, \mathbf{s}_{-i}^{*}\right)+\eta$. Note that when a user deviates, it does not care about the reliability of the other users but only her own reliability. So in the above definitions $\left(s_{i}, \mathbf{s}_{-i}^{*}\right)$ is not necessarily $(1-\epsilon)$-reliable.

Given any $\bar{\epsilon}>0$, the capacity region $\mathcal{C}$ of the interference channel is the closure of the set of all rate vectors $\left(R_{1}, \ldots, R_{K}\right)$ such that for every $\epsilon \in(0, \bar{\epsilon})$, there exists a $(1-\epsilon)$-reliable strategy profile $\mathbf{s}$ that achieves the rate vector $\left(R_{1}, \ldots, R_{K}\right)$. The Nash equilibrium region $\mathcal{C}_{\mathrm{NE}}$ of the interference channel is the closure of the set of rate pairs $\left(R_{1}, \ldots, R_{K}\right)$ such that for every $\eta>0$, there exists a $\bar{\epsilon}>0$ (dependent on $\eta$ ) so that if $\epsilon \in(0, \bar{\epsilon})$, there exists a $(1-\epsilon)$-reliable strategy profile $\mathbf{s}$ that achieves the rate vector $\left(R_{1}, \ldots, R_{K}\right)$ and is a $\eta$-NE. Clearly, $\mathcal{C}_{\mathrm{NE}} \subseteq \mathcal{C}$.

Following [8], we consider $\mathcal{C}_{\mathrm{NE}}$ in the context of the linear deterministic interference channel model introduced in [2]. In this channel, channel input for each is interpreted as a succession of levels: $x=0 . b_{1} b_{2} b_{3} b_{4} b_{5} \ldots$ each representing one bit of the real-valued input to the corresponding Gaussian channel. The most significant bit coincides with the highest level, the least significant bit with the lowest level. Noise is modeled by truncation. Bits of smaller order than the noise are lost. The signal from transmitter $i$, as observed at receiver $j$, is scaled by a nonnegative integer gain $a_{j i}=2^{n_{j i}}$ (equivalently, the input column vector is shifted up by $n_{j i}$ ). At each time $t$, the input and output, respectively, at link $i$ are $\mathbf{x}_{i}(t), \mathbf{y}_{i}(t) \in\{0,1\}^{q}$, where $q=\max _{i j} n_{i j}$. Note that $n_{i i}$ corresponds to $\log _{2} \mathrm{SNR}_{i}$ and $n_{j i}$ corresponds to $\log _{2} \mathrm{INR}_{j i}$, where $\mathrm{SNR}_{i}$ is the signal-to-noise ratio of link $i$ and $\mathrm{INR}_{j i}$ is the interference-to-noise ratio at receiver $j$ from transmitter $i$ in the corresponding Gaussian interference channel. To model the super-position of signals at each receiver, the bits received on each level are added modulo two. The channel output at receiver $i$ is then given by

$$
\mathbf{y}_{i}(t)=\sum_{j=1}^{K} \mathbf{S}^{q-n_{i j}} \mathbf{x}_{j}(t),
$$

where summation and multiplication are in the binary field and $\mathbf{S}$ is a $q \times q$ shift matrix (e.g. see [5]). For several symmetric examples considered in this paper, the direct gains $n_{i i}$ will be denoted by $n_{d}$, whereas, the cross-gains $n_{i j}$ will be denoted by $n_{c}$.

\footnotetext{
${ }^{1}$ We use the notation $\mathbf{s}_{-i}$ to denote the set of strategy choices for every user except user $i$. Also, with a slight abuse of notation we use $\left(s_{i}, \mathbf{s}_{-i}\right)$ to denote the profile $\mathbf{s}$.

${ }^{2}$ In the game theoretic literature, this is often referred to as an $\epsilon$-Nash equilibrium or simply an $\epsilon$-equilibrium for a game [12, page 143].
} 

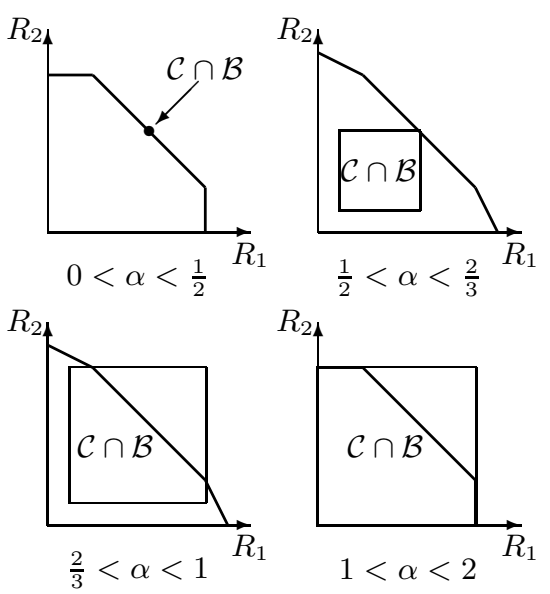

Fig. 1. Examples of $\mathcal{C}_{\mathrm{NE}}$ for a symmetric deterministic interference channel with normalized cross gain $\alpha$.

In [8], $\mathcal{C}_{\mathrm{NE}}$ was completely characterized for the linear deterministic channel model with $K=2$ users. In particular, $\mathcal{C}_{\mathrm{NE}}$ was shown to be equal to the intersection of a "box" $\mathcal{B}$ and the capacity region $\mathcal{C}$ of the deterministic channel (see Figure 1). The intersection is always non-empty and contains at least one point on the sum-rate boundary of $\mathcal{C}$.

It was shown in [8] and [9] that sum-rate optimal NEs for 2 -user and some special $K$-user cases can be attained by using very simple 'structured strategies' which involve doing one of the following three options on each signal level:

- Transmit an independent bit to be decoded by the receiver

- Transmit a 'copy' bit, i.e., transmit the same bit that is transmitted from some other level

- Transmit noise, i.e., a random bit that is not intended to be decoded by the receiver

\section{INDICES}

As can be seen from Fig. 1, in general $\mathcal{C}_{\mathrm{NE}}$ will contain multiple points, not all of which obtain the same sum-rate. In this section we examine two indices to characterize the best and worst case efficiency that can be obtained at a Nash equilibrium, where efficiency is measured relative to the sumcapacity $R_{\text {sum }}^{C}$. Specifically, we define

$$
\begin{aligned}
& \text { - } \gamma_{\max }=\frac{\left(R_{\text {sum }}^{N E}\right)_{\max }}{R_{\text {sum }}^{C}} \\
& \text { - } \gamma_{\min }=\frac{\left(R_{\text {sum }}^{N E}\right)_{\min }}{R_{\text {sum }}^{C}}
\end{aligned}
$$

where $R_{\text {sum }}^{N E}$ is the sum-rate of the users in a given NE. The first index $\gamma_{\max }$ measures the best-case scenario in terms of the equilibrium sum-rate. For a given interference channel, it is not possible to achieve an efficiency greater than $\gamma_{\max }$ in $\mathcal{C}_{\mathrm{NE}}$. This index is essentially the inverse of the 'price of stability' metric studied in the algorithmic game theory literature [15]. The second index, $\gamma_{\min }$ measures the worst-case scenario in terms of sum-rate when the players reach an NE in the interference game. If the players are in an NE, the efficiency in terms of sum-rate cannot go below this value. This index is again the inverse of the so-called 'price of anarchy'.
Below we compute these index values for a number of symmetric cases for which we know the sum-capacity. In each of these symmetric channels all users have the same direct gain $n_{d}$ and between any pair of users the cross gain is either 0 or $n_{c}=\alpha n_{d}$.

Before we consider the indices for the different cases, we shall prove a simple lemma that will be used to evaluate $\gamma_{\min }$.

Lemma 1: In any linear deterministic interference channel, the 'worst-case' NE is achieved by adopting the following strategy: Transmit independent bits from levels which are intereference-free at a user's own receiver. Transmit noise from all other levels so as to create as much interference as possible at other receivers.

Proof: First, note that such a strategy will be an equilibrium as no player can improve their rate. A lower informationrate can only be achieved by not transmitting an independent bit from a level corresponding to an interference-free level at the receiver. But such a strategy cannot be an equilibrium as the same player can increase her pay-off by transmitting an independent bit from that level.

\section{A. 2-user deterministic interference channel}

The entire NE region for the 2-user interference channel has been characterized in [8]. For the symmetric case, the $\gamma_{\max }$ and $\gamma_{\min }$ can be readily evaluated by looking at Figure 1 and the capacity region of a 2-user linear deterministic IC. Since, for all values of $\alpha$, there exists an NE that is on the sumcapacity boundary,

$$
\gamma_{\max }=1
$$

For $\alpha<\frac{1}{2}$, this is the unique NE. In the region $\frac{1}{2}<\alpha<1$, the lower left-hand corner of the 'box-bound' approches the origin and then stays there for all values of $\alpha>1$. Hence, it can be shown that

$$
\gamma_{\min }= \begin{cases}1, & 0 \leq \alpha \leq 1 / 2 \\ \frac{1-\alpha}{\alpha}, & 1 / 2 \leq \alpha \leq 2 / 3 \\ \frac{1-\alpha}{1-\frac{\alpha}{2}}, & 2 / 3 \leq \alpha \leq 1 \\ 0, & 1 \leq \alpha\end{cases}
$$

\section{B. K-user Many-to-One deterministic interference channel}

In [9] it was shown that $\mathcal{C}_{\mathrm{NE}}=\mathcal{B} \cap \mathcal{C}$. Further, it was shown that $\mathcal{C}_{\mathrm{NE}}$ always contains a sum-rate optimal rate point. For the symmetric case that we consider here, for $\alpha<1$, there is only one NE. For $\alpha>1$, due to the fact that all the users interfering with the common user (say, user 1) can use the levels below their own noise floor to transmit noise, the 'anarchy' drives the $\gamma_{\min }$ to values below 1 . Thus for all values of $\alpha$, we have

$$
\gamma_{\max }=1
$$

while,

$$
\gamma_{\text {min }}= \begin{cases}1, & 0 \leq \alpha \leq 1 \\ \frac{1}{1+\frac{\alpha-1}{K-1}}, & 1 \leq \alpha \leq 2 \\ \frac{K-1}{K}, & 2 \leq \alpha\end{cases}
$$

However, as $K \rightarrow \infty, \gamma_{\min } \rightarrow 1$. 


\section{K-user One-to-Many deterministic interference channel}

This channel, often viewed as complementary to the manyto-one channel discussed before, exhibits an important characteristic that it may not have a sum-rate optimal NE. It was also shown in [9] that for $K>2$ and $n_{i 1}>n_{11}$ for at least two users $i \neq 1, \mathcal{C}_{\mathrm{NE}} \neq \mathcal{B} \cap \mathcal{C}$. Further $\mathcal{C}_{\mathrm{NE}}$ is not guaranteed to contain a sum-rate optimal point. In a symmetric case, we can characterize this loss of efficiency as follows:

$$
\gamma_{\max }= \begin{cases}\frac{K(1-\alpha)+\alpha}{K-\alpha}, & 0 \leq \alpha \leq 1 \\ \frac{K(\alpha-1)+(2-\alpha)}{K-(2-\alpha)}, & 1 \leq \alpha \leq 2 \\ 1, & 2 \leq \alpha\end{cases}
$$

Likewise, using lemma 1, we have

$$
\gamma_{\min }= \begin{cases}\frac{K(1-\alpha)+\alpha}{K-\alpha}, & 0 \leq \alpha \leq 1 \\ \frac{1}{K-(2-\alpha)}, & 1 \leq \alpha \leq 2 \\ \frac{1}{K}, & 2 \leq \alpha\end{cases}
$$

Note that, as $K \rightarrow \infty$, for $\alpha>1, \gamma_{\min } \rightarrow 0$.

\section{K-user bi-symmetric deterministic interference channel}

A 3-user bi-symmetric interference channel was introduced in [9]. This is a special case of a more general 'shoe-string' interference channel whose sum-capacity was characterized in [14]. Here, we consider a symmetric $K$-user extension of this channel where $K-1$ users interfere with user 1 , while user 1 also interferes with all the other $K-1$ users. None of these $K-1$ users interfere with each other. Using the sum-capacity results of [14] and using the symmetry in interference, it can be shown that, for this channel,

$$
\mathcal{C}_{\text {sum }}= \begin{cases}K n_{d}-2 \alpha n_{d}, & 0 \leq \alpha \leq 1 / 2 \\ (K-1) n_{d}, & 1 / 2 \leq \alpha \leq 1 \\ (K-1) n_{d}+(\alpha-1) n_{d}, & 1 \leq \alpha \leq 2 \\ K n_{d}, & 2 \leq \alpha\end{cases}
$$

In [9], it was shown that for a 3-user bisymmetric interference channel, $\mathcal{C}_{\mathrm{NE}}$ contains a sum-rate optimal point for $\alpha>2 / 3$. Using the same proof technique we can prove a similar claim. Further, we can characterize the efficencies of the NEs as follows:

$$
\begin{gathered}
\gamma_{\max }= \begin{cases}\frac{K(1-\alpha)}{K-2 \alpha}, & 0 \leq \alpha \leq 1 / 2, \\
\frac{K \alpha}{K-1}, & 1 / 2 \leq \alpha \leq 2 / 3, \\
1, & 2 / 3 \leq \alpha .\end{cases} \\
\gamma_{\text {min }}= \begin{cases}\frac{K(1-\alpha)}{K-2 \alpha}, & 0 \leq \alpha \leq 1 / 2, \\
\frac{K(1-\alpha)}{K-1}, & 1 / 2 \leq \alpha \leq 1, \\
0, & 1 \leq \alpha .\end{cases}
\end{gathered}
$$

Note that, unlike the $K$-user one-to-many case where $\gamma_{\text {min }} \rightarrow$ 0 asymptotically in $K$ for $\alpha>1$, the worst case efficiency can be 0 for even $K=3$.

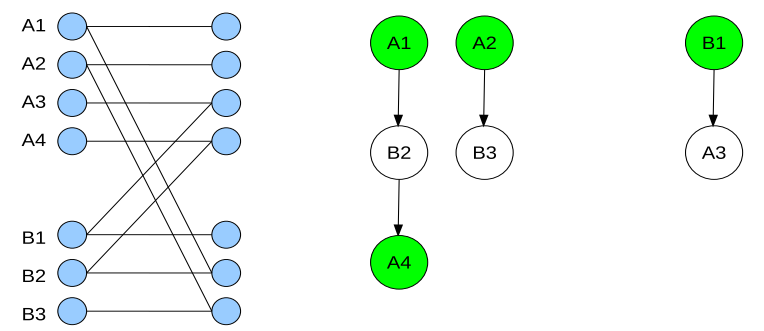

$$
n_{11}=4, n_{12}=2, n_{21}=2, n_{22}=3 \begin{aligned}
& \cdot \text { Transmit and receive levels are merged into a single node } \\
& \cdot \text { Direction of arrow shows interference } \\
& \cdot \text { Independent bits are transmitted from each green node } \\
& \cdot \text { No transmission is attempted from white nodes }
\end{aligned}
$$

Fig. 2. NE strategy when cost of transmission is included in pay-off

\section{Games with a Cost of Transmission}

The low values of $\gamma_{\text {min }}$ for several channel models in the previous section arise due to the fact that there exists such equilibria where users unnecessarily transmit noise from levels below their own receiver's noise floor, thereby reducing reliable transmission rate for other users. A reasonable way to discourage this sort of 'harmful' behavior is to associate a 'cost' to transmissions. This implies that a user's strategy will include transmission from a particular level only when that transmission contributes to a gain in her reliable bit-rate, and not otherwise. We define a new 'interference game' is similar to the game introduced in Section II, but with a new pay-off function for user $i$, given by

$$
P_{i}=R_{i}-t \cdot T_{i}
$$

where a cost of $t<1$ is incurred due use of each level, and $T_{i}$ is the total number of levels that user $i$ uses for transmission. For the present discussion, we restrict ourselves to a game where the players are allowed to adopt strategies that either involve transmission of an independent bit from a level, send a copy or not transmit anything at all. We let $\mathcal{G}_{p}$ denote this modifed game. Note that, the strategies that achieve $\gamma_{\max }$ in all the previous examples belong to this strategy space.

Lemma 2: For the 2-user linear deterministic interference channel $\mathcal{G}_{p}$ has a unique NE for each parameter tuple.

Proof: First, note that under the new pay-off scheme, sending a copy bit from a level cannot be part of an equilibrium strategy because, by not transmitting the copy the user can increase her pay-off due to savings in terms of the cost of transmission. Similarly, sending noise from level's below a user's own receiver's noise floor cannot be part of a equilibrium strategy. Thus, in the chosen strategy space, any equilibrium strategy must have the following characterization:: Each user transmits independent bits only from levels which do not receive any interference from the other user.

Given the knowledge of the channel parameters, any such $\mathrm{NE}$ can be found by creating an 'interference graph' as described in [13]. An equilibrium is then given by choosing 


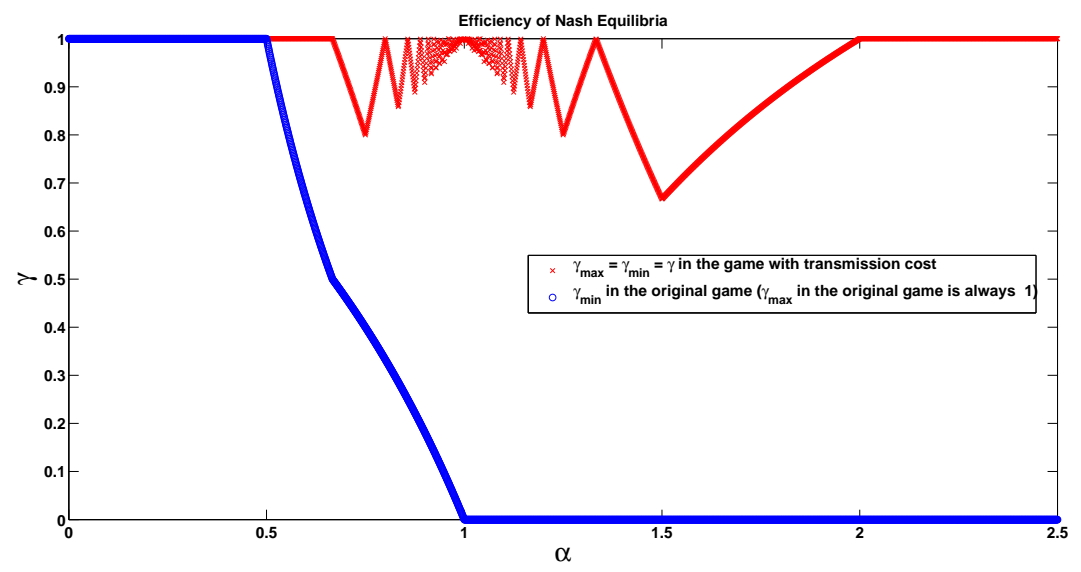

Fig. 3. Efficiency of 2-user Nash Equilibria with different pay-offs

alternate levels (starting from the top-most level of each chain) to transmit an independent bit. This is illustrated in Figure 2. In case of cycles (which can only be composed of two nodes), the choice of levels is not unique, but the sum-rate achieved is. Such a situation arises, for example in the symmetric case $\alpha=1$ which has $\left(\begin{array}{c}2 n_{d} \\ n_{d}\end{array}\right)$ strategy pairs, all yielding the same sum-rate of $n_{d}$.

Inclusion of the cost of transmission in the pay-off function enables us to get rid of many inefficient equilibria and thereby increase the value of $\gamma_{\min }$ for all parameter values. However, for this two user case, $\gamma_{\max }$ is no longer guaranteed to be always 1. Figure 3 compares the efficiencies with and without the cost of transmission.

Lemma 3: The uniqueness (upto sum-rate) of NE is not guaranteed in a $K$-user interference network where $K>2$

Proof: Consider a 3-user bi-symmetric interference channel introduced in [9]. Suppose $\alpha=1$. There are two different Nash equilibria - one in which only the middle user transmits from all her levels achieving a sum-rate of $n_{d}$ while other two users do not transmit at all, and another in which both the fringe users transmit from all their levels while the middle user does not transmit at all, thus achieving the sum-capacity of $2 n_{d}$.

Note that for both the $K$-user symmetric 'one-to-many' and 'many-to-one' interference networks, the new pay-off function ensures existence of a sum-rate unique NE. The proof follows by drawing 'interference graphs' and noting the absence of any cycle in the respective graphs.

\section{CONCLUSIONS}

In this paper we compared the efficiencies (with respect to sum-capacity) of the different Nash equilibria for interference games in several $K$-user symmetric linear deterministic channels. We also introduced a new pay-off function that includes a 'cost of transmission'. With this new game and a restricted set of strategies, we proved that for two users there exists a unique Nash Equilibrium. Investigating the new game in the context of 'unrestricted' strategy sets and also for more general $K$ user intereference networks is left as future work.

\section{REFERENCES}

[1] R. Etkin, D. Tse, and H. Wang, "Gaussian Interference Channel Capacity to within One Bit," IEEE Trans. on Information Theory, Vol. 54, No. 12, Dec. 2008.

[2] G. Bresler and D. Tse, "The Two-User Gaussian Interference Channel: A Deterministic View," European Transactions in Telecommunications, vol. 19, pp. 333-354, April 2008.

[3] G. Bresler, A. Parekh, and D. Tse, "The Approximate Capacity of the many-to-one and one-to-many Gaussian interference channel," Allerton Conference on Communication, Control and Computing, Monticello, IL, Sept. 2007.

[4] A. El Gamal and M. Costa, "The Capacity Region of a Class of Deterministic Interference Channels," IEEE Transactions on Information Theory, Vol. IT-28, No. 2, pp. 343-346, March 1982.

[5] S. Avestimehr, S. Diggavi, and D. Tse, "Wireless Network Information Flow," Allerton Conference on Communication, Control, and Computing, Monticello, IL, September 2007.

[6] R. Etkin, A. P. Parekh and D.Tse, "Spectrum Sharing in Unlicensed Bands", IEEE Journal on Selected Areas of Communication, vol. 25, no. 3, pp. 517-528, April 2007.

[7] S. T. Chung, S. J. Kim, J. Lee, and J.M. Cioffi, "A game-theoretic approach to power allocation in frequency-selective Gaussian interference channels," Proceedings of IEEE ISIT, pp. 316-316, June 2003.

[8] R. Berry and D. Tse, "Information Theoretic Games on Interference Channels," Proceeding of IEEE ISIT, pp. 2518-2522, July 2008.

[9] R. Berry and S. Saha, "On Information Theoretic Interference Games with More than Two Users," Proceedings of IEEE Information Theory Workshop, Cairo, Egypt, January, 2010.

[10] R. Berry and D. Tse, "Shannon Meets Nash on the Interference Channel," submitted to IEEE Transactions on Information Theory, May 2010.

[11] X. Liu and E. Erkip, "Alternating-Offer Bargaining Games over the Gaussian Interference Channel,"Proceedings of Forty-Eighth Annual Allerton Conference on Communication, Control, and Computing, SepOct 2010.

[12] R. Myserson, Game Theory: Analysis of conflict, Harvard University Press, Cambridge MA, 1991.

[13] S. Saha and R. Berry, "On the Combinatorial Structure of 2-user Linear Deterministic Interference Channel and Better Response Updates,"Presented as a Poster at ISIT 2010, Austin, TX, June, 2010.

[14] S. Saha and R. Berry, "On the Sum-capacity of a Class of 3-user Deterministic Interference Channels,"Proceedings of Forty-Eighth Annual Allerton Conference on Communication, Control, and Computing, SepOct 2010.

[15] N. Nisan et, al., "Algorithmic Game Theory", Cambridge University Press, 2007. 